\title{
TROP-2 is a novel reliable marker for immunohistochemical diagnosis of papillary thyroid carcinoma
}

\author{
Andrey Bychkov, Pichet Sampatanukul, Shanop Shuangshoti, Somboon Keelawat \\ Department of Pathology, Faculty of Medicine, Chulalongkorn University, Bangkok, Thailand
}

\section{Introduction and Objective}

so Immunohistochemistry (IHC) in thyroid pathology is recruited for differentiating between benign and malignant tumors

80 There is no single antibody that can render definitive diagnosis

\&o Most of the recently identified IHC markers have never been assimilated into daily clinical practice due to their low reproducibility and other limitations

\&o TROP-2 IHC was recently reported as a useful tool for identifying PTC in fine-needle aspirate and tissue microarray samples

so We aimed to validate utility of TROP-2 immunostaining in the differential diagnosis of thyroid tumors

\section{Materials and Methods}

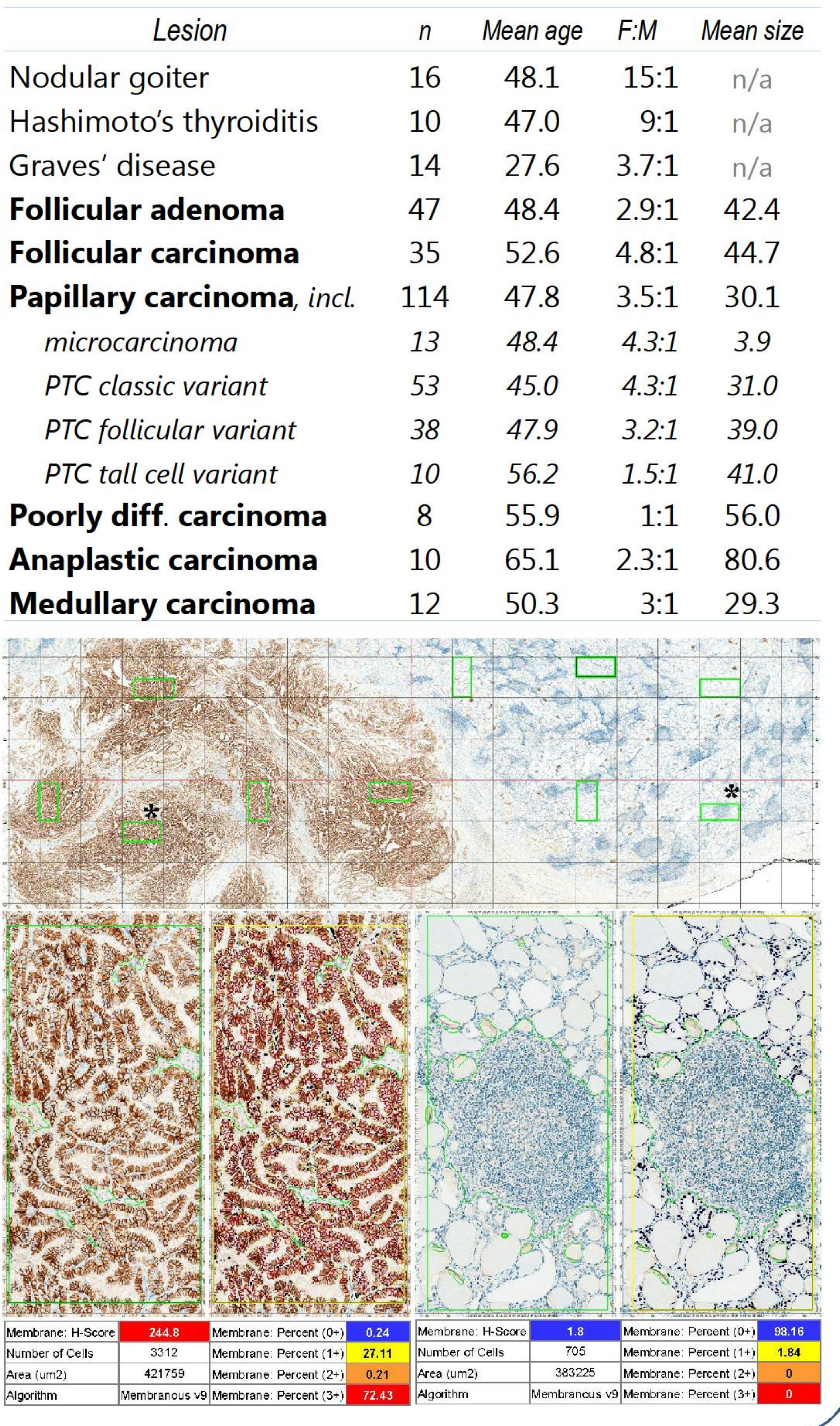

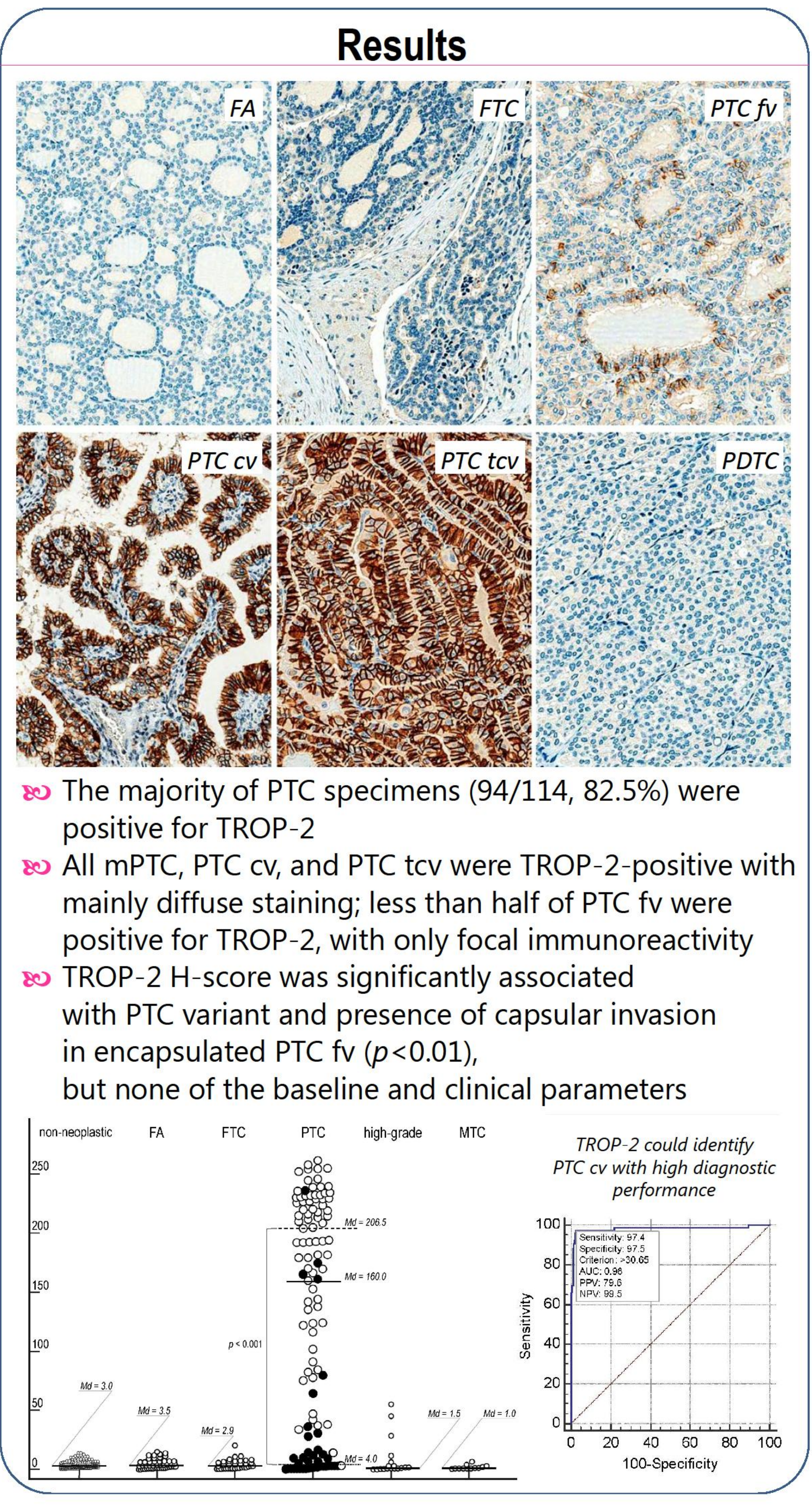

\section{Conclusions}

80 TROP-2 membranous staining is a very sensitive and specific marker for PTC cv, PTC tcv, and MPTC - with high overall specificity for PTC

80 More than $10 \%$ of TROP-2 positive cells in a section, regardless of intensity of membranous staining supports a diagnosis of PTC

80 TROP-2 is recommended for use in differential diagnosis of benign and malignant thyroid nodules 